\title{
Efficient Approximation of Edit Distance
}

\author{
Robert Krauthgamer* \\ Faculty of Mathematics and Computer Science \\ Weizmann Institute of Science, Rehovot, Israel \\ robert.krauthgamer@weizmann.ac.il
}

\begin{abstract}
The similarity between two strings is often measured by some variant of edit distance, depending on the intended application. But even the basic version of this distance, which is simply the minimum number of character insertions, deletions and substitutions needed to transform one string to the other, presents remarkable algorithmic challenges.

This talk will examine the task of approximating the basic edit distance between two strings, starting with the classical RAM model and moving on to computational models which impose further constraints, such as the query complexity model and the sketching model. Beyond their concrete applications, these investigations provide a wealth of information about the problem, teaching us state of the art techniques and uncovering the limitations of certain methodologies. We will then come full circle with improved algorithms for the classical RAM model. During this journey, we may encounter special cases like permutation strings, whose patterns are all distinct, and smoothed instances, which are a mixture of worst-case and average-case inputs.

Finally, we shall discuss known gaps and open problems in the area, including variants of the basic edit distance, such as allowing block moves, or edit distance between trees, and hopefully touch upon related computational problems like nearest-neighbor search.
\end{abstract}

The original version of this chapter was revised: The copyright line was incorrect. This has been corrected. The Erratum to this chapter is available at DOI: $10.1007 / 978-3-319-02432-5 \_33$

* Work was supported in part by the Israel Science Foundation (grant \#897/13), the US-Israel BSF (grant \#2010418), and by the Citi Foundation.

O. Kurland, M. Lewenstein, and E. Porat (Eds.): SPIRE 2013, LNCS 8214, p. 3, 2013.

(C) Springer-Verlag Berlin Heidelberg 2013 